\title{
La influencia de las nuevas tecnologías en la profesión: el traductor en la era de internet
}

\author{
Tanagua Barceló Martínez \\ Universidad de Málaga \\ tbmartinez@uma.es \\ Francisca García Luque \\ Universidad de Málaga \\ paquigar@uma.es
}

Resumen: Este trabajo pretende describir cuáles son las principales modificaciones que internet ha introducido en el quehacer de traductores e intérpretes, desde su etapa formativa hasta su vida laboral. Para ello, haremos un breve recorrido histórico para dar cuenta de otros hitos que han marcado la historia de estas dos profesiones hasta desembocar en las últimas décadas del siglo XX, profundamente marcadas por la irrupción de este fenómeno.

Palabras clave: traducción; interpretación; internet; nuevas tecnologías.

\section{The influence of new technologies in the profession: The translator in the} Internet era

Abstract: This paper aims to describe the main transformations introduced by Internet in the daily work of translators and interpreters, ranging from their instruction period to their professional life. To this end, we will review briefly the main milestones in the history of these two professions to culminate in the last decades of the $20^{\text {th }}$ century, deeply marked by the emergence of this phenomenon.

Keywords: translation; interpreting; Internet; new technologies. 



\section{Introducción}

A lo largo de la historia, hallamos referencias a las primeras traducciones de textos sagrados o de textos literarios, los considerados canónicos. Durante la Edad Media, parte de la cultura occidental nos llegó a través de la labor de los traductores de los monasterios. El descubrimiento de América también supuso una revolución para una tarea que tiene mucho que ver con las culturas y con las diferentes concepciones del mundo que entran en contacto en la pluma del traductor. Más tarde, surgieron traducciones de textos de naturaleza más variada (jurídica, económica, científica o audiovisual). Los cambios en el entorno de trabajo y en el concepto mismo de la traducción han ido de la mano de la evolución de la sociedad. Pero la transformación más importante que nos ha tocado vivir en un pasado reciente es la llegada de internet y de las nuevas tecnologías.

Este trabajo pretende describir cuál ha sido la influencia que la llegada de internet ha tenido en la profesión del traductor. Haremos también referencia a la profesión del intérprete, en la medida en que hay características que afectan a ambas. No se trata de analizar programas o herramientas concretas sino más bien de una reflexión sobre una influencia que se materializa a distintos niveles, desde la formación al quehacer diario de los traductores, pasando por el acceso a la documentación, el nacimiento de nuevas modalidades de traducción íntimamente relacionadas con la tecnología o las posibilidades de acceso al mercado de trabajo.

\section{Hitos en la historia de la traducción}

La traducción y la interpretación son dos profesiones que han visto cómo sus caminos se han ido cruzando a lo largo de la historia. Actualmente, la primera cuenta con una sólida base mientras que la segunda se desarrolla a veces a la sombra de los paradigmas creados bajo el paraguas de la Traductología. Según indica Van Hoof (1999: 7-18), las primeras fuentes documentales sobre estas profesiones nos llevan al antiguo Egipto, unos 3000 años a. C., cuando unas inscripciones halladas en Asuán dan cuenta de la figura del intérprete, que contaba con un jeroglífico específico y que constituía una verdadera casta privilegiada al servicio del faraón. Estos príncipes de Elefantina transmitían su oficio 
de padres a hijos. No es, por tanto, descabellado pensar que traducción e interpretación coexistieron en esta civilización que mantenía contactos con tantos pueblos limítrofes. Los primeros testimonios escritos que confirman una actividad traductora datan del tercer milenio a. C., con el hallazgo de unas tablillas en la biblioteca real de Nínive, que contienen gramáticas y glosarios trilingües en las lenguas sumeria, acadia y babilónica.

Conocida es también la existencia de traductores e intérpretes en la civilización griega y en el Imperio romano, dedicados a las campañas militares, el comercio o la administración. Aunque los testimonios de los que disponemos atañen fundamentalmente a la literatura, es fácil imaginar que hubo otros tipos de textos objeto de traducción, relacionados con la vida cotidiana. Un caso especial son los textos religiosos, con los que comienza la reflexión sobre la actividad traductora y las necesidades de la traducción del Nuevo Testamento.

El primer traductor occidental de una obra literaria que dejó su nombre para la posteridad fue Livio Andrónico, traductor de obras como $L a$ Odisea de Homero. Por entonces, las figuras del autor y el traductor no estaban claramente diferenciadas y el hecho de redactar una obra en una lengua, aun proviniendo de otra, otorgaba cierto grado de originalidad. La civilización romana fue la responsable de traducir al latín el legado de la antigua Grecia, y de esta época nos han llegado algunas consideraciones relacionadas con los principios y métodos para traducir, de la mano de Cicerón, quien se cuestionaba ya la eterna dicotomía entre la fidelidad a la forma y la fidelidad al sentido. Más tarde, la hegemonía islámica supo beber de la sabiduría de los pueblos conquistados a través de la traducción, haciendo que su civilización brillase por encima de estos.

Pero si hay un hito en la historia de la traducción a lo largo de la Edad Media es la Escuela de Traductores de Toledo, que aunque no fue un lugar geográfico al que se dirigían sabios de todos los rincones de Europa (Santoyo, 2008: 85-6), sí constituye un núcleo de actividad traductora cuya influencia para el saber y la ciencia es innegable. A través de los traductores de la época, la Europa cristiana pudo conocer los avances científicos provenientes del mundo árabe. Según Catelli y Gargatelli (1998), los traductores medievales, cuyos nombres ya conocemos, desarrollaron una amplia labor y se caracterizaron por ser muy literales, ante las dificultades que hallaban a la hora de interpretar textos que les resultaban oscuros. 
Otra época que marcó profundamente la labor del traductor como mediador lingüístico $\mathrm{y}$, sobre todo, como transmisor de cultura, fue la que sobrevino tras el descubrimiento de América. Con este acontecimiento, los pueblos conquistadores iniciaron una labor de aculturación de las civilizaciones indígenas americanas en la que la traducción de todo tipo de textos sirvió para imponer unos valores y una visión del mundo que pasó necesariamente por las manos de los traductores. Es sintomático que la actividad traductora tuviese lugar solo desde el español hacia las lenguas indígenas. Catelli y Gargatelli (ibidem) ven en este hecho un deseo deliberado de ocultar al otro.

Avanzando un poco más en el tiempo, entramos en la Edad Moderna, cuando resulta más fácil hallar rastros escritos de traducciones y traductores, gracias a la extensión del uso de la imprenta y el asentamiento del concepto de autoría, distinto del de traducción. Esta actividad comienza a extenderse y a salir del ámbito religioso y literario para introducirse en los ámbitos comercial y administrativo. Así empieza a desempeñar un papel importante en el ámbito económico, además del religioso, el literario y el político. Con la llegada del siglo XVIII aumenta la labor de traducción, que empieza a ser fundamental en la difusión de todas las disciplinas científicas. Los siglos XIX y XX continúan con la expansión de esa actividad, que culmina en la actualidad con nuestra "aldea global", en la que la sociedad de la información, la globalización, la desaparición de las barreras comerciales y la intensificación de la comunicación producen el volumen de traducción y de interpretación más intenso de toda la historia. No en vano, es en el pasado siglo cuando surgen la interpretación consecutiva, en la conferencia de paz de París, y la interpretación simultánea, durante el proceso de Nuremberg (Baigorri, 2000). Ambas surgen como respuesta a una realidad geopolítica nueva y ante la que era necesario hallar una respuesta que asegurase la viabilidad de una comunicación efectiva. A partir de entonces, la interpretación en todas sus modalidades está presente en casi todos los ámbitos de la sociedad.

Nos hallamos, por tanto, ante una labor, la de traductores e intérpretes, que se ha ido adaptando y redefiniendo con el paso del tiempo, mientras ha ido penetrando cada vez en más ámbitos de la sociedad, hasta impregnar hoy día casi todos los terrenos en los que podemos pensar. No es de extrañar que Delisle y Woodsworth (2007) califiquen a los traductores a lo largo de la historia como inventores de alfabetos, 
constructores de lenguas nacionales, artífices de las literaturas nacionales, difusores del conocimiento, actores en los escenarios de poder, propagadores de religiones, importadores de valores culturales y redactores de diccionarios, para rematar definiendo a los intérpretes como testigos privilegiados de la historia.

Tras este repaso por algunos de los acontecimientos que más han influido a la hora de modelar la profesión del traductor e intérprete, nos centraremos en la descripción del rasgo distintivo que constituye el núcleo de interés de este trabajo, internet y su influencia en nuestra profesión en las últimas décadas. Trataremos de dibujar una panorámica general que dé cuenta del impacto que la llegada de internet ha supuesto en la vida de los traductores e intérpretes.

\section{Surgimiento de internet}

Internet ha supuesto una verdadera revolución en la vida diaria de los ciudadanos de nuestro tiempo. Este hito tecnológico se puede definir, de acuerdo con Rouse (2014), del siguiente modo:

The Internet, sometimes called simply "the Net", is a worldwide system of computer networks - a network of networks in which users at any one computer can, if they have permission, get information from any other computer (and sometimes talk directly to users at other computers).

En un principio, internet surge en el ámbito militar, como respuesta a los desafíos de Rusia durante la Guerra Fría. E1 7 de abril de 1969 está considerada la fecha oficial de la creación de Internet, en el momento en el que se consiguió conectar entre sí dos ordenadores a distancia, aunque la verdadera comunicación entre ellos no se produjo hasta unos meses después, en octubre del mismo año. En 1986, asistimos a la implantación de los nombres de dominio, lo que dio paso al registro de dominios propios por parte de las empresas. Esto propició la proliferación de información disponible en la red, hasta llegar a la incontrolable cantidad de información presente actualmente en ella.

En la década de los noventa, internet irrumpe con fuerza en el día a día de los particulares y, por extensión, en el de traductores e intérpretes. En apenas veinticinco años hemos comprobado cómo su quehacer diario se ha visto transformado debido a la existencia de dicha 
herramienta y de todo lo que ella ha traído consigo. Y esto a diferentes niveles: acceso a la información; búsqueda documental; recepción y envío de materiales; herramientas de ayuda a la traducción; contacto con clientes y profesionales; formas de pago; etc. En palabras de Gonzalo García (2000: 145),

[...] puede advertirse que desde hace algún tiempo los modos tradicionales de practicar la traducción se han venido transformando de forma notable a consecuencia de la irrupción y generalización de los nuevos recursos tecnológicos de apoyo a la comunicación interlingüística.

En la actualidad, la presencia y el uso de internet en nuestras vidas supera cualquier predicción que hubiese podido hacerse $\mathrm{y}$, comparativamente, el desarrollo y el impacto de esta revolución tecnológica han sido mucho más rápidos que los de otros grandes inventos o descubrimientos y ha venido a cambiar las formas de trabajo e introducir modificaciones en los sistemas de enseñanza, en las comunicaciones profesionales y sociales. En este sentido, compartimos la reflexión de Eco (2000) cuando afirma que:

Il computer e Internet sono la vera rivoluzione del secolo, che può modificare, come a suo tempo la stampa, il nostro modo di pensare e di apprendere. L'invenzione della stampa ha prodotto la libera interpretazione della Bibbia, e ha fatto sorgere una nuova pedagogia fondata su libri illustrati che insegnavano l'alfabeto (vedi Comenio). Prima che esistesse la stampa, un bambino non poteva aver accesso a un manoscritto. Oggi, grazie a Internet, possiamo sapere cose che i nostri antenati impiegavano una vita a conoscere.

Al hablar de internet y de sus repercusiones en la vida del traductor, debemos distinguir dos grandes etapas, denominadas web 1.0, por un lado, y web 2.0 o web social, por otro. Esta última supuso, "the big change of paradigm in the web" (Vargas Sierra y Ramírez Polo, 2011). Este cambio radical en la red también supuso una transformación para la actividad traductora. Así, los traductores pueden (ibidem)

[...] interact, collaborate and share by means of social media in a virtual community where everybody could contribute. Examples of these types of applications include blogs, wikis, video sharing sites, social networks, web applications and web services, among others. 
La labor del traductor, basada en la comunicación en su sentido más amplio, ha sido partícipe de este cambio radical y, en pocos años, se ha visto completamente transformada. Este cambio no solo afecta a la actividad profesional del traductor, sino también a las etapas formativas previas a su incorporación al mercado de trabajo. En el siguiente apartado trataremos de poner de relieve los cambios más significativos que el traductor ha vivido en los últimos años como consecuencia del surgimiento de Internet.

\section{Internet en la vida de traductores e intérpretes}

A continuación, vamos a desarrollar cuáles son las principales modificaciones que caracterizan la labor de traductores e intérpretes en la actualidad como consecuencia de la irrupción de internet en nuestras vidas, de acuerdo con la idea apuntada por Somers (2003: 13) de que "[...] the existence and growth of Internet, could be said to have revolutionised the job of the translator". Dado que esta influencia se manifiesta en varios momentos y en varios niveles, vamos a dividir este apartado en diversos bloques. El primero de ellos hace referencia a la etapa formativa, en la que los cambios han sido más que notables. El segundo se adentra ya en el ejercicio profesional de la traducción y la interpretación, en el que abordaremos, a su vez, distintas perspectivas.

Antes de intentar analizar cuáles han sido estos cambios, conviene detenerse, aunque tan solo sea de forma somera, en cuáles son las habilidades que se van a exigir a traductores e intérpretes y que les van a permitir acometer su trabajo con el mayor rigor. El conjunto de dichas habilidades es lo que conocemos como competencia traductora, definida por Kelly (2002: 9) como el:

conjunto de capacidades, destrezas, conocimientos e incluso actitudes que reúnen los traductores profesionales y que intervienen en la traducción como actividad experta, es decir que, en su conjunto, distinguen al profesional del no profesional, al experto del no experto.

Dentro de la competencia traductora, otro referente en el ámbito de la didáctica de la traducción como es el Grupo PACTE distingue las siguientes subcompetencias:

1. Competencia comunicativa en dos lenguas

2. Competencia extralingüística 
3. Competencia instrumental/profesional

4. Competencia de transferencia

5. Competencia estratégica

6. Competencias psicofisiológicas

En el presente trabajo nos centraremos en la competencia instrumental/ profesional, que es la que guarda mayor relación con nuestro trabajo, por englobar todo aquello que tiene que ver con el uso de la tecnología a la hora de traducir. Parece claro que, actualmente, el manejo de estas herramientas es condición sine qua non para el ejercicio de la profesión de traductor e intérprete. Y con el objeto de que dicho manejo sea lo más profundo y amplio posible, es necesario iniciar el aprendizaje desde la formación del futuro traductor, es decir, resulta primordial incluir en los planes de estudio universitarios programas que permitan desarrollar esta subcompetencia. Tomando, pues, como base estas premisas pasamos a continuación a describir cómo se materializa la influencia de internet en los distintos niveles y etapas aludidos anteriormente.

\subsection{En la etapa formativa}

La aparición de internet ha provocado, por las razones ya mencionadas, la modificación de los planes de estudios en los grados de Traducción e Interpretación, que han introducido toda una serie de materias relacionadas con el uso de las tecnologías y con la adquisición de la subcompetencia instrumental/profesional. A este respecto, Diéguez y Lazo (2004) afirman que:

La implementación de las TIC en la enseñanza universitaria requerirá, a juicio de Cabero (2002), un alumno más preocupado del proceso que del producto, preparado para la toma de decisiones y que sepa elegir su ruta de aprendizaje, es decir, debe estar preparado para el autoaprendizaje. Lo anterior implica un cambio en el trabajo del profesor, quien debe transformarse en un diseñador de situaciones instruccionales para el alumno, tutor del proceso didáctico, facilitador y consejero sobre fuentes apropiadas de información y creador de hábitos y destrezas en la búsqueda, selección y tratamiento de la información.

En España, la Agencia Nacional de Evaluación de la Calidad y Acreditación (ANECA) distingue, en el Libro Blanco del título de Grado en 
Traducción e Interpretación, entre competencias transversales o genéricas (entre las que destacan los conocimientos de informática aplicada, diseño y gestión de proyectos), por un lado; y, por otro, las competencias específicas: dominio de lenguas extranjeras, conocimiento de culturas y civilizaciones extranjeras, dominio de la lengua propia, escrita y oral, dominio de técnicas y terminología de la traducción especializada, manejo de herramientas informáticas, dominio de técnicas de traducción asistida/localización, destreza para la búsqueda de información/ documentación, conocimiento de los aspectos económicos y profesionales, capacidad de trabajo en equipo, capacidad de diseñar y gestionar proyectos, poseer una amplia cultura.

De entre las competencias específicas mencionadas, tres (manejo de herramientas informáticas, dominio de técnicas de traducción asistida/ localización, destreza para la búsqueda de información/documentación) están íntimamente relacionadas con el uso y el manejo de internet y, en lo tocante al resto de competencias, la red supone una fuente de información y consulta que resulta también de vital importancia actualmente, ya que las complementa y las potencia.

En las conclusiones incluidas en el Libro Blanco (a las que se ha llegado tras el análisis de las encuestas realizadas a estudiantes, profesionales y empleadores del ámbito de la traducción y la interpretación), la $\operatorname{ANECA~(2004:~110)~señala~que~}$

la mayoría [de los estudiantes, profesionales y empleadores] tiene en mente a un traductor generalista, de amplia base, muy bien formado en todo lo que se refiere a las lenguas y culturas de su elección, mientras un sector importante destaca la necesidad de contar con sólidas destrezas y conocimientos de informática y con la capacidad de crear y coordinar proyectos de traducción. Ambos insisten en que hay que adquirir y mejorar la competencia en idiomas e informática y la competencia cultural, algo que, probablemente, se convierte en un objetivo permanente de su vida profesional.

Asimismo, el Libro Blanco incluye una serie de contenidos mínimos que deberían constar en todos los planes de estudio del título de Traducción e Interpretación en la universidad española. Dentro de estos contenidos, los relativos a la traducción hacen mención expresa a la "excelencia en el manejo de las herramientas informáticas de apoyo a la traducción". Y, con respecto a los contenidos instrumentales, se alude 
específicamente a las "técnicas de investigación documental; fuentes, metodología y gestión terminológicas; tecnologías aplicadas a la traducción y la interpretación".

Por todo lo expuesto, la totalidad de los grados de Traducción e Interpretación implantados en España con motivo de la llegada del Espacio Europeo de Educación Superior cuenta con varias asignaturas que han sido concebidas con el objetivo de que los alumnos adquieran esta subcompetencia, en la que internet desempeña un papel crucial. En el caso concreto de la Universidad de Málaga, esta implantación se materializa de manera específica en tres asignaturas: Recursos informáticos aplicados a la Traducción e Interpretación, Herramientas para la práctica de la Traducción I: Terminología y Herramientas para la práctica de la Traducción II: Documentación. Además, las competencias relacionadas con el uso de internet se hallan igualmente presentes en aquellas asignaturas dedicadas a la traducción especializada, en sus diversas ramas (jurídica y socioeconómica, científico-técnica, humanística $\mathrm{y}$ audiovisual), al igual que en las asignaturas de interpretación, en sus distintas modalidades (bilateral, consecutiva y simultánea). Todo ello en la medida en que el proceso de documentación, realizado fundamentalmente a través la red, es una de las etapas fundamentales de la labor de los traductores e intérpretes en formación.

La llegada de estas nuevas asignaturas a los planes de estudio y la modificación de las ya existentes no ha supuesto solo una modificación de estos sino que, además, obliga a docentes y discentes a actualizar constantemente sus conocimientos para adaptarse a la principal característica de internet, que es el ritmo vertiginoso al que evoluciona y se transforma. En esta misma línea, las instituciones educativas, fundamentalmente las universidades, han debido adaptar sus infraestructuras, en un sentido amplio, a las nuevas exigencias que esta nueva realidad plantea. Hacemos referencia con esto, por ejemplo, a la proliferación de las aulas de informática, la creación de servicios de laboratorios tecnológicos, la creación de plataformas virtuales de enseñanza, la posibilidad de conexión permanente a la red desde los distintos espacios universitarios, etc. Aunque todos estos recursos son aplicables a la totalidad de los estudios universitarios, estamos en condiciones de afirmar que los estudios de Traducción e Interpretación se sirven especialmente de ellos para acercar la realidad profesional a la etapa formativa. 


\subsection{En la profesión}

\subsubsection{Transformaciones generales en el ejercicio profesional}

No corremos el riesgo de equivocarnos al afirmar que las cosas han cambiado mucho desde que Kay describiera su visión de los ordenadores como "translator's amanuensis" (1980: 12), es decir, una especie de herramienta, más o menos sofisticada, de la que el traductor podía hacer uso para realizar determinadas tareas y que habría de evolucionar para satisfacer sus necesidades. No obstante, parece obvio afirmar que hombre y máquina viven actualmente en simbiosis en el mercado profesional de la traducción. De acuerdo también con Somers (op. cit.), casi desde el inicio de las investigaciones en traducción automática asomaba la idea de que más que sustituir al hombre, la máquina aspiraba a convertirse en una ayuda.

Según indica Hutchins (1997), el uso de estas herramientas de ayuda a la traducción se generalizó a partir de los años 90, en el momento en que a los desarrollos tecnológicos anteriores se añadió la mayor capacidad de almacenamiento de los ordenadores. De acuerdo con este autor, estas nuevas herramientas, entre las que menciona las bases de datos terminológicas y diccionarios, los glosarios y recursos terminológicos, los procesadores de textos multilingües o los instrumentos que permiten flexibilizar la gestión de los textos (formatos, transmisión o maquetación) entre otros, "[...] offer translators the opportunity of making their work more productive without taking away the intelectual challenge of translation".

Todas las nuevas oportunidades que se presentan gracias o a través de internet han llevado a distinguir a Vargas Sierra y Ramírez Polo (op. cit.) entre dos tipos de usuarios: los usuarios pasivos y los usuarios activos. Los primeros "deploy these tools to be connected with other professionals and to be updated about the news on the field", mientras que los segundos "not only consume information, but also participate in creating content within these new social media".

Haciendo pues un rápido repaso de las muchas herramientas que el desarrollo tecnológico y el nacimiento de internet han traído consigo, partimos de la clasificación realizada por Cid Leal y Recoder Sellarés (2000) y vamos a enumerar aquellas que, a nuestro parecer, han tenido 
una mayor repercusión para la traducción, independientemente del ámbito o campo.

- Listas de distribución: medios de comunicación basados en el correo electrónico a los que se accede mediante suscripción individual. La proliferación de listas de distribución de correo en el ámbito de la traducción ha sido notable en los últimos años. Es un instrumento que permite recabar una información puntual de un número muy amplio de posibles destinatarios con una inversión mínima en tiempo y esfuerzo, ya que se envía un único mensaje, lo que permite rentabilizar la gestión del tiempo dedicado a la documentación y a la consulta.

- Blogs: definidos por la Real Academia de la Lengua Española como un "sitio web que incluye, a modo de diario personal de su autor o autores, contenidos de su interés, actualizados con frecuencia y a menudo comentados por los lectores". Al igual que en el caso anterior, la proliferación de blogs de traducción e interpretación es un fenómeno muy visible en la red. La acumulación de experiencias, datos e información útil en este formato tampoco es en absoluto desdeñable.

- Foros: que se entienden como una plataforma virtual que permite el encuentro, el intercambio de información y la publicación de mensajes con el objetivo de compartir datos, experiencias, dudas, etc. En el caso de la traducción, de nuevo es un formato que cuenta con una notable presencia en internet.

- Herramientas de ayuda a la traducción: aplicaciones basadas en memorias de traducción que se ubican en la red y que están a disposición de los usuarios.

Todas estas herramientas, algunas de las cuales no son de uso exclusivo del ámbito de la traducción, cobran una especial relevancia en nuestro campo, y se sitúan en la línea del trabajo colaborativo, un concepto muy en boga en nuestro actual sistema educativo, que, en este sentido, adopta un enfoque constructivista. Pero este sistema de trabajo no es exclusivo del ámbito formativo sino que caracteriza en gran medida la actividad profesional del traductor, tal y como comentaremos más adelante. 


\subsubsection{Transformaciones en los ámbitos de especialidad}

A continuación, nos centraremos en describir aquellos tipos de recursos que, gracias a la red, han modificado especialmente la forma de trabajar de traductores e intérpretes dentro de los diferentes ámbitos de especialidad.

En los ámbitos jurídico, económico, científico y técnico, fundamentalmente, son de especial interés los siguientes recursos:

- Bases de datos: herramienta electrónica que se basa en el almacenamiento sistemático y ordenado de datos dentro de un mismo contexto. Merecen una especial mención aquellas bases de datos puestas a disposición del usuario por organismos internacionales como la ONU o la Unión Europea, por citar algunas especialmente representativas.

- Repositorios textuales: una variante de la anterior herramienta basada en el almacenamiento de textos, lo que permite al traductor realizar tareas relacionadas con la macroestructura textual, las convenciones de género, convenciones ortotipográficas, búsquedas terminológicas, etc.

- Obras de corte lexicológico o terminológico: especialmente diccionarios, glosarios, léxicos y tesauros, disponibles en línea.

- Revistas y publicaciones especializadas de acceso en línea: que permiten al traductor, por una parte, ampliar los conocimientos temáticos y, por otra, conocer el estado de la investigación o la evolución de algún aspecto puntual relacionado con su ámbito de trabajo.

- Herramientas para trabajar con corpus: herramientas que, a partir de la compilación previa de un corpus textual, permiten llevar a cabo análisis de distinta naturaleza (terminológica, estilística, de tipo colocacional, etc.) con el objeto de definir las características del corpus en cuestión y obtener conclusiones sólidas.

En lo que respecta a la traducción audiovisual, que se caracteriza no por el campo temático sino por el formato en el que se desarrolla, se le podría aplicar todo lo dicho hasta ahora, en la medida en que el contenido lo exija, y añadir los siguientes cambios, relativos al formato. 
- La cercanía geográfica de los traductores con respecto a los estudios de doblaje y subtitulado. En la actualidad, ya no es necesario que el traductor resida cerca del estudio para el que trabaja a la hora de recibir el documento audiovisual. Internet ha abierto la puerta a otras posibilidades: el envío a través del e-mail, el uso de plataformas para compartir contenidos, por ejemplo Dropbox, o incluso el alojamiento de los contenidos audiovisuales en la denominada "nube" y el acceso al traductor mediante una clave.

- La aparición de nuevas modalidades de TAV, como los fansubs y los fandubs, que son traducciones hechas por y para aficionados y disponibles en línea.

Después de este breve repaso, pasaremos a analizar de manera sucinta cuáles han sido las principales consecuencias que estas nuevas herramientas y este nuevo contexto han traído consigo.

El traductor ha visto ampliados tanto los modos de llevar a cabo su trabajo como las posibilidades de crecimiento profesional debido a la creación de nuevos perfiles profesionales fruto del auge de las nuevas tecnologías. Con respecto a lo primero, el traductor ha visto reforzada la posibilidad de convertirse en un profesional liberal, lo que ha fomentado el aumento considerable de traductores autónomos, quienes, además, cuentan con la ventaja de poder residir y trabajar tan lejos de sus clientes como deseen, y también con todos los posibles inconvenientes que ello pueda suponer, como señalan Cid Leal y Recoder Sellarés (op. cit.):

Los traductores son uno de los colectivos profesionales que más rápidamente han accedido al teletrabajo. Para muchos, esto representa una ventaja inmensa, puesto que pueden situar su despacho profesional en un pueblo perdido entre montañas o en la ciudad más bulliciosa sin salir de casa, siempre y cuando estén conectados a Internet y dispongan de un buen equipo informático. Pero a su vez, y como cualquier teletrabajador, pueden sentirse demasiado aislados de su entorno social y laboral $[\ldots]$

Con respecto al surgimiento de nuevos perfiles profesionales, podemos destacar la figura del gestor de proyectos, indispensable a la hora de coordinar, a través de la red, el volumen de trabajo de todos los profesionales que colaboran en un determinado proyecto, a menudo en 
diferentes localizaciones geográficas. En palabras de Vargas Sierra y Ramírez Polo (op. cit.),

examples of these new profiles include pre-editors and post-editors (reviewers of machine translation input and output), linguistic consultants, quality assurance experts, and machine experts, including the development of skills involved in designing, operating and maintaining natural language processing machines etc.

Esta transformación en el ámbito de la traducción ha llevado aparejado el beneficio, por así decirlo, para determinados sectores profesionales del ámbito de las tecnologías y, en principio, ajenos a la traducción. Esto tiene que ver con las nuevas herramientas que traductores e intérpretes utilizan actualmente y que llevan a estos sectores a la creación y desarrollo de programas de ayuda a la traducción y la interpretación con sus constantes actualizaciones (desde un programa de traducción asistida hasta una cabina de interpretación dotada de su correspondiente programa informático).

La realidad actual, caracterizada por la constante aparición y actualización de herramientas y aplicaciones tecnológicas (ordenadores, tabletas, teléfonos móviles, videojuegos, etc.), no solo ha modificado la forma de trabajar del traductor sino que, además, ha tenido un gran impacto sobre los "géneros" textuales que conforman el volumen de trabajo de los profesionales. Nos referimos especialmente a la localización de todo tipo de productos informáticos, desde programas informáticos o videojuegos hasta programas para cajeros automáticos, productos de navegación de medios de transporte, traducción de páginas webs y productos audiovisuales, aplicaciones para teléfonos móviles, etc.

\section{Reflexión final}

Todos los cambios a los que hemos aludido generan una reflexión en torno a las múltiples ventajas y las posibles desventajas que llevan aparejados.

Con respecto a las primeras, no cabe duda de que la aparición de internet ha permitido al traductor ampliar su abanico de posibilidades a la hora de trabajar, y ello desde distintas perspectivas: el acceso a la documentación y a los distintos recursos, el modo de recibir y enviar 
trabajo, su ubicación geográfica, su posibilidad de movilidad, su independencia, su cartera de clientes, etc.

Con respecto a las segundas, cabría destacar el aumento de la invisibilidad, la soledad del traductor, la dependencia que las tecnologías crean con respecto al contacto con clientes e intermediarios, la desconfianza hacia los recursos tradicionales (diccionarios en papel), el fomento del anonimato y el aumento de las exigencias en cuanto al manejo de herramientas de todo tipo por parte de los clientes o empleadores, lo que obliga a un reciclaje continuo.

\section{Bibliografía}

Agencia Nacional de Evaluación de la Calidad y Acreditación. 2004. Libro blanco del título de Grado en Traducción e Interpretación. www.aneca. es/media/150288/libroblanco_traduc_def.pdf [Acceso 18/01/2016].

Baigorri Jalón, Jesús. 2000. La interpretación de conferencias: el nacimiento de una profesión. Granada: Comares, Colección Interlingua.

Catelli, Nora \& Gargatagli, Marieta. 1998. El tabaco que fumaba Plinio: escenas de la traducción en España y América, relatos, leyes y reflexiones sobre los otros. Barcelona: Ediciones del Seibel.

Cid, P. y Recoder, M. J. 2000. Dónde están las llaves... o los recursos digitales para la traducción. En Fuentes, E. (dir.) Anuari de biblioteconomia i informació: Bibliodoc 2000 = Anuario de biblioteconomía i documentación = Library and information sciences yearbook. Barcelona: Col·legi Oficial de Bibliotecaris-Documentalistes de Catalunya, 51-68.

Delisle, Jean \& Woodsworth, Judith (dirs.) 2007. Les traducteurs dans l'histoire. Ottawa: Presses de l'Université d'Ottawa.

Diéguez, M. I. \& Lazo, R .M. 2004. Tecnologías de la información y de la comunicación (TIC) al servicio del traductor profesional. Onomazéin 9 1: 5174. https://repositorio.uc.cl/bitstream/handle/11534/7866/000367179. pdf? sequence=1\&isAllowed=y [Acceso 05/03/2016].

Eco, Umberto. 2000 (8 de enero). La cultura corre online, chi non si adegua è perduto. La Repubblica. http://ricerca.repubblica.it/repubblica/archivio/repubblica/2000/01/08/eco-la-cultura-corre-on-line-chi.html [Consulta: $10 / 03 / 2016]$.

Fernández Polo, Francisco Javier. 2005. Internet en la formación del traductor. En Varela, M. C. (coord.) Nuevas tecnologías en lingüistica, traducción y enseñanza de lenguas. Universidad de Santiago de Compostela: Servicio de Publicaciones, 109-132. 
Gonzalo García, Consuelo. 2000. Fuentes lingüísticas en Internet para el traductor. En Gonzalo García, Consuelo \& García Yebra, Valentín (eds.) Documentación, terminología y traducción. Madrid: Editorial Síntesis, 145-166.

Hutchins, John. 1997. Translation Technology and The Translator. En Greensmith, Catherine \& Vandamme, Marilyn (ed.) Proceedings [of] International conference, exhibition \& AGM. London: Institute of Translation and Interpreting, 113-120.

Kay, Martin. 1980/1997. The proper place of men and machines in language translation. Machine Translation. 12(1-2): 3-23

Kelly, Dorothy A. 2002. La competencia traductora: bases para el diseño curricular. Puentes 1: 7-20.

PACTE. 2001. La competencia traductora y su adquisición. Quaderns. Revista de Traducció 6: 39-45. http://ddd.uab.cat/pub/quaderns/11385790n6/11 385790n6p39.pdf [Acceso 01/03/2016].

Rouse, Margaret. 2016. Techtarget. http://searchwindevelopment.techtarget. com/definition/Internet [Acceso 10/02/2016].

Somers, Harold (ed.). 2003. Computers and Translation. A Translator's Guide. Benjamins Translation Library. Amsterdam/Philadelphia: John Benjamins Publishing Company.

Van Hoof, Henri. 1991. Histoire de la traduction en Occident. Paris-Louvainla-Neuve: Duculot.

Vargas Sierra, Chelo \& Ramírez Polo, Laura. 2011. The Translator's Workstations revisited: A new paradigm of translators, technology and translation. Ponencia presentada en el congreso "Tralogy. Futures in Techonolgies for Translation. Anticiper les tecnologies pour la traduction". Paris: 3-4 marzo 2011. http://lodel.irevues.inist.fr/tralogy/index. php?id=71 [Acceso 11/10/2016].

Santoyo, Julio César. 1999. Historia de la traducción: quince apuntes. León: Ediciones Universidad de León, Secretariado de Publicaciones. 